Chirurg 2012 $\cdot 83: 738$

DOI 10.1007/s00104-012-2345-3

Online publiziert: 22. Juli 2012

(c) Springer-Verlag 2012
T. Franzke · J. Jähne

Klinik für Allgemein- und Viszeralchirurgie, Schwerpunkt für endokrine und

onkologische Chirurgie, Diakoniekrankenhaus Henriettenstiftung, Hannover

\title{
Laparoskopisch assistierte vs. offene Gastrektomie bei fortgeschrittenen Magenkarzinomen
}

\section{Eine Fall-Kontroll-Studie}

\section{Orginalpublikation \\ Kim KH, Kim MC, Jung GJ et al (2012) Com- parative analysis of five-year survival results of laparoscopy-assisted gastrectomy versus open gastrectomy for advanced gastric can- cer: a case-control study using a propensity score method. Dig Surg 29:165-171}

Die meisten Studien zur laparoskopisch assistierten Gastrektomie beziehen sich auf frühe Tumorstadien. Die vorliegende Arbeit soll die Machbarkeit der laparoskopisch assistierten Operation (inklusive D2-Lymphadenktomie) sowie auch das onkologische Outcome von Patienten mit fortgeschritten Magenkarzinomen im Vergleich zum offenen Vorgehen zeigen.

\section{Methodik}

In einer Fall-Kontroll-Studie („single center") wurden 434 Patienten retrospektiv ausgewertet, die zwischen 1999 und 2007 aufgrund eines fortgeschrittenen Magenkarzinoms ( $\geq \mathrm{pT} 2, \mathrm{~N} 1, \mathrm{M} 0)$ gastrektomiert wurden (laparoskopisch assistierte Gastrektomie [LAG], $\mathrm{n}=89$; konventionelle Gastrektomie [OG], n=345). In beide Gruppen wurden letztlich 88 Patienten einbezogen. Alle Patienten wurden mit 6 Zyklen 5-FU und Cisplatin neoadjuvant behandelt. Das postoperative Follow-up betrug 5 Jahre. Einschlusskriterien waren unter anderen die R0-Resektion sowie die Durchführung einer D2-Lymphadenektomie.

\section{Ergebnisse}

Die mittlere Operationsdauer war bei der LAG signifikant länger als bei der OG $(228,3 \pm 49,4 \mathrm{~min}$ vs. $183,6 \pm 42,7 \mathrm{~min}$, $\mathrm{p}<0,0001)$. Die Anzahl der entfernten Lymphknoten zeigte keinen signifikanten Unterschied zwischen beiden Gruppen $(38,3$ vs. $41,8, p=0,1187)$. In beiden Gruppen lag die Komplikationsrate bei $8 \%(n=7)$, wobei in der LAG-Gruppe 2 $(2,2 \%)$ und in der OG-Gruppe eine $(1,1 \%)$ Major-Komplikation (Anastomoseninsuffizienz) auftrat. Die Zeit bis zum ersten Stuhlgang wie auch die Krankenhausverweildauer war in der LAG-Gruppe signifikant kürzer (3,2 vs. 3,7 Tage, $\mathrm{p}<0,0001$; $7,0$ vs. 10,4 Tage, $\mathrm{p}<0,0001)$. Während des Follow-ups zeigte die Rezidivrate $(14,8$ vs. $17,1 \%, p=0,8371)$ keinen signifikanten Unterschied ( 14,8 vs. $17,1 \%, \mathrm{p}=0,8371)$. Die 5-Jahres-Überlebensraten betrugen 85,9 und $83,1 \%(\mathrm{p}=0,4630)$.

\section{Diskussion und Fazit}

Die vorliegende Studie belegt die Machbarkeit der laparoskopisch assistierten Gastrektomie inklusive der D2-Lymphadektomie durch erfahrene Chirurgen auch bei fortgeschrittenen Karzinomen. Hinsichtlich des postoperativen Verlaufs konnten die für die laparoskopische Chirurgie allgemein bekannten Vorteile auch für die LAG gezeigt und ähnliche Überlebensraten wie beim offenen Vorgehen erzielt werden. Allerdings weist die Studie deutliche methodische Schwächen („single center", retrospektiv, nicht randomisiert) auf. Die Tatsache, dass aus der LAG-Gruppe fast alle Patienten, aus der OG-Gruppe aber nur ein vergleichsweise geringer Teil der Patienten in die FallKontroll-Studie eingeflossen sind, deutet auf einen erheblichen Bias hin. Darüber hinaus überraschen die sehr guten 5-Jahres-Überlebensraten. Trotz dieser nicht unerheblichen Einschränkungen unterstützt diese Studie den Trend, dass auch große onkologische Oberbaucheingriffe vermehrt laparoskopisch durchgeführt werden. Eine generelle Empfehlung zur laparoskopisch assistierten Gastrektomie kann allerdings keinesfalls ausgesprochen werden.

\section{Korrespondenzadresse}

\section{Dr. T. Franzke}

Klinik für Allgemein- und Viszeralchirurgie, Schwerpunkt für endokrine und onkologische Chirurgie, Diakoniekrankenhaus Henriettenstiftung, Marienstr. 72-90, 30171 Hannover Timm.Franzke@ddh-gruppe.de

Interessenkonflikt. Der korrespondierende Autor gibt für sich und seinen Koautor an, dass kein Interessenkonflikt besteht. 